Schweizerisches Jahrbuch für Entwicklungspolitik

18 | 1999

Die Schweiz und die humanitäre Aktion

\title{
Humanitäre Hilfe, Sicherheitspolitik und Krisenprävention
}

Günther Baechler

\section{OpenEdition}

1 Journals

Electronic version

URL: http://journals.openedition.org/sjep/630

DOI: 10.4000/sjep.630

ISSN: 1663-9677

Publisher

Institut de hautes études internationales et du développement

Printed version

Date of publication: 1 janvier 1999

Number of pages: $39-49$

ISSN: 1660-5926

\section{Electronic reference}

Günther Baechler, « Humanitäre Hilfe, Sicherheitspolitik und Krisenprävention », Schweizerisches

Jahrbuch für Entwicklungspolitik [Online], 18| 1999, Online erschienen am: 18 Juli 2012, abgerufen am 08 September 2020. URL : http://journals.openedition.org/sjep/630 ; DOI : https://doi.org/10.4000/ sjep.630

(c) The Graduate Institute 


\section{HUMANITÄRE HILFE, SICHERHEITSPOLITIK UND KRISENPRÄVENTION}

GÜNTHER BAECHLER*

\section{KONFLIKTE UND HUMANITÄRE KATASTROPHEN AN DER SCHWELLE ZUM NÄCHSTEN JAHRHUNDERT}

Ende der neunziger Jahre setzt sich weltweit ein Trend fort, der bereits nach dem Ende des Zweiten Weltkriegs erkennbar war, seit der Überwindung des Kalten Kriegs aber immer klarere Konturen annimmt: das Kriegsgeschehen verlagert sich immer mehr in das Innere von Entwicklungsländern. So fanden nach 1989 in rund einem Drittel sämtlicher Entwicklungsländer - einschliesslich der neuen unabhängigen Staaten Zentralasiens bewaffnete Auseinandersetzungen statt. Betrachtet man nur die Gruppe der ärmsten Länder, dann war sogar die Hälfte von akuten Konflikten betroffen. Hinzu kommt eine begrenzte und insgesamt abnehmende Zahl bewaffneter Konflikte im Kaukasus, im ehemals unter sowjetischer Herrschaft stehenden Osteuropa und im Balkan. Die fast ausschliesslich innerstaatlichen Kriege aller genannten Regionen sind Ausdruck von tiefgreifenden gesellschaftlichen Umbrüchen und daher oft langwierig, ökonomisch zerstörerisch, politisch schwer kalkulierbar und mit grossem menschlichem Leid verbunden. ${ }^{1}$

Der von Gewalt begleitete soziopolitische Wandel in Entwicklungs- und Transitionsländern hat Konsequenzen für die Entwicklungspolitik und die humanitäre Hilfe.

Einerseits: Der stärkste Gegner der Entwicklungszusammenarbeit ist der Bürgerkrieg. Entwicklungszusammenarbeit droht unter Bedingungen verall-gemeinerter Gewalt zur kurzfristigen Katastrophenhilfe zu werden. Friedensförderung dient oft mehr der Nachkriegsstabilisierung als der Krisen- und Gewaltprävention. Ein immer grösser werdender Anteil der Mittel für Entwicklungszusammenarbeit fliesst in die Behebung von Kriegsfolgeschäden. Zivilbevölkerungen sind die hauptsächlichen Opfer von ethnopolitisch, nationalistisch und macht bzw. verteilungspolitisch motivierter Gewalt. Gemäss einer Aufstellung des Schweizerischen Katastrophenhilfekorps (SKH) stieg zwischen 1983 und 1993 die Zahl der laufenden bewaffneten Konflikte von 39 auf 55. Allerdings stieg im gleichen Zeitraum auch die Zahl von Naturkatastrophen, nämlich von 50 auf 130, wobei sich Kriegsfolgen und Naturkatastrophen nicht in jedem Fall voneinander trennen lassen. ${ }^{2}$ Die Ausgaben der OECD-Staaten für bilaterale Nothilfe bei Kriegsfolgen und Naturkatastrophen stiegen von 1980 bis 1992 von $2 \%$ auf $6,3 \%$ der weltweiten öffentlichen Entwicklungszusammenarbeit dieser Länder; im Jahr 1994 waren es bereits 10\%. Nach 1994 ist der Trend allerdings

* Direktor, Schweizerische Friedenstiftung, Institut für Konfliktlösung, Bern.

1. Baechler G. FAST: Frühanalyse von Spannungen und Tatsachenermittlung. Abschlussbericht zum Pilotprojekt FAST. Working paper 26, Schweizerische Friedensstiftung. Institut für Konfliktlösung, Bern 1997, S. 8.

2. Eine Welt, Nr. 2, Juni 1998, S.6. 
wieder leicht rückläufig. In konkreten Zahlen heisst das: die OECD-Staaten gaben 1988 rund 500 Mio. US\$ für humanitäre Hilfe aus und 5 Jahre später (1993) bereits siebenmal mehr, nämlich 3,5 Mrd. ${ }^{3}$ Wie in einem vom deutschen Bundesministerium für wirtschaftliche Zusammenarbeit (BMZ) vorgelegten Bericht beklagt wird, «werden [damit] der Entwicklungszusammenarbeit die Mittel für ihre eigentlichen Aufgaben entzogen, nämlich langfristig Strukturen für eine nachhaltige, sich selbst tragende Entwicklung aufzubauen ». ${ }^{4}$

Andererseits : Der Mitteltransfer von langfristiger Entwicklungszusammenarbeit im Sinne struktureller Krisenvorsorge hin zu relativ kurzfristiger humanitärer Not- und Wiederaufbauhilfe ist nur die eine Seite der Medaille. Die andere Seite ergibt sich aus noch kostspieligeren «humanitären Interventionen» im Sinne von Friedensoperationen der Vereinten Nationen (peacekeeping). Ein vorläufiger Höhepunkt bildete das Jahr 1994, als insgesamt 78'000 Personen beim peacekeeping der Vereinten Nationen zum Einsatz kamen. Die Kosten dafür beliefen sich auf rund 4 Mrd.\$. ${ }^{5}$ In diesem Jahr kamen kriegsbedingt mehr als 4 Mio. Menschen um, während sich nach Berechnungen des UNHCR rund 27 Mio. Menschen auf der Flucht befanden.

Humanitäre Aktionen, das heisst sowohl Hilfe als auch Interventionen, mussten in erster Linie dort durchgeführt werden, wo lokale bewaffnete Konflikte, Bürgerkriege, ethnopolitische Krisen und Genozide soziale, wirtschaftliche und politische Aktivitäten behinderten. Hilfe und Intervention mussten somit in einem sicherheitspolitisch schwierigen, wenn nicht prekären Umfeld geleistet werden. Für beide Aspekte humanitärer Aktionen hatte dies im Laufe der neunziger Jahre Konsequenzen, jedoch auf unterschiedliche Weise.

Im folgenden wird argumentiert, dass gerade aufgrund der gegenläufigen Tendenzen im peacekeeping einerseits und der humanitären Hilfe andererseits letztere noch stärker als bisher sicherheitspolitischen Zwängen unterworfen ist. Um dies zu illustrieren, werden zuerst die internationalen Entwicklungen beleuchtet. Vor diesem Hintergrund wird auf die Diskussion in der Schweiz - insbesondere im Kontext des «Brunner-Berichts» - eingegangen. Zum Schluss wird der Akzent auf die Krisenprävention gelegt, denn nur vorbeugende Politik vermag letztlich den zunehmenden Druck auf die Nothilfe zu mindern.

\section{LEID LINDERN UND FRIEDEN SICHERN:}

VON DER HUMANITÄREN LEGITIMIERUNG DER SICHERHEITSPOLITIK ZUR FRIEDENSPOLITISCHEN BEGRÜNDUNG DER HUMANITÄREN HILFE

\section{$\square$ Auf- und Abstieg humanitärer Interventionen in den neunziger Jahren}

Die humanitäre Intervention fand ihren Höhepunkt und ihre grösste Vielfalt zur Zeit der UNPROFOR 1994/95 im früheren Jugoslawien. Durch eine teils gewagte Kombination von zivilen und militärischen Interventionen in innerstaatliche Krisen sollte das Leid von Zivilbevölkerungen gelindert und eine

3. Bundesministerium für Zusammenarbeit (Hrsg.) BMZ aktuell: Entwicklungszusammenarbeit und Krisenvorbeugung, Bonn, Juni 1997, S. 2.

4. Schmieg E. Entwicklungszusammenarbeit und Krisenvorbeugung, Bonn 1995 (Manus.), S. 2.

5. Die Kosten für die 18 UN Missionen im Jahr zuvor beliefen sich auf 3.6 Mrd. US\$. Vgl. Lund M.S. Preventing Violent Conflicts. A Strategy for Preventive Diplomacy, Washington D.C. 1996, S. 22 f. 
Bedrohung des Weltfriedens gemäss Kapitel VII der Charta der Vereinten Nationen abgewandt werden. Entscheidungen des Sicherheitsrates erfuhren durch die zivil-militärischen Interventionen immer mehr eine humanitäre Begründung. $\mathrm{Zu}$ den unterschiedlichen Interventionsformen gehörten kostenund personalintensive Einsätze, wie

\ das Monitoring oder die Durchführung von Wahlen in nicht völlig befriedeten Gebieten oder Ländern, wie zum Beispiel in Angola oder Kambodscha;

a der Schutz von Einwohnern einer Region, ob Minderheiten oder Mehrheit, gegen drohende Gewaltanwendung, so zum Beispiel die drei sogenannten United Nations Protected Areas (UNPA) in Kroatien oder der «safe heaven » zum Schutz der Kurden im Norden Iraks;

व die Errichtung und die Kontrolle über «safe areas», etwa in bosnischen Städten, gegen militärische An- und Übergriffe ;

- Absicherung der (teilweisen) Entmilitarisierung von bestimmten Zonen, etwa um Sarajewo und Goradze in Bosnien;

- Sicherstellung des Transports und der Verteilung von humanitären Hilfsgütern sowie weiterer humanitärer Massnahmen (Gesundheit, Gefangene, psychosoziale Dienste) während der heissen Phase eines Konflikts, wie in grossem Massstab in Somalia geschehen;

a Unterstützung der Wiederherstellung der Ordnungsfunktion einer Regierung (Polizei) nach einem Bürgerkrieg, etwa in El Salvador oder Kambodscha;

a Monitoring und Berichterstattung über die Verletzung des humanitären Völkerrechts durch die Kriegsparteien sowie von Menschenrechten während und nach einem Krieg (Rwanda). ${ }^{6}$

Nach der Hochphase im Jahr 1995 nahmen zwar nicht die Anzahl, jedoch Grösse, Kosten und Komplexität der Missionen rasch und deutlich ab. Ende Dezember 1997 waren weniger als 15’000 Personen, das heisst Militär und Zivilpolizei, im Einsatz. Die Kosten beliefen sich gerade noch auf 1.3 Mrd. US\$. Zum Vergleich: Alleine in Moçambique beliefen sich nach dem Ende des Bürgerkriegs die Kosten für die Rückführung von Flüchtlingen, die Demobilisierung der Streitkräfte, den Wiederaufbau der Infrastruktur und die Unterstützung des Staatshaushalts von 1992 bis 1995 auf geschätzte 2 Mrd. US\$.

Aufgrund solcher und vergleichbar komplexer Missionen mit einer in Erfolge und Misserfolge geteilten Bilanz sah sich der Sicherheitsrat der Vereinten Nationen dazu gezwungen, die Planung und Durchführung grösserer Operationen zurückzustellen oder aufzugeben (Burundi, Demokratische Republik Kongo). Heute werden kleinere und einfachere Missionen bevorzugt.

Die einzig neue Mission, die der Sicherheitsrat 1997 beschloss, war die United Nations Verification Mission in Guatemala (MINUGUA). Diese verbindet in beispielgebender Weise ein humanitäres mit einem sicherheitspolitischen Mandat, ohne die Grenzen zwischen den beiden zu verwischen. Einer zunächst reinen Menschenrechtsmission wurden vorübergehend für drei Monate 155

6. Roberts A. «The Crisis in UN Peacekeeping». In: Crocker C.A. et al. Managing Global Chaos. Sources of and Responses to International Conflict, Washington D.C. 1996, S. 297-319.

7. Ibid. 
Militärbeobachter beigefügt. Diese hatten die begrenzte Aufgabe, die Erfüllung der Oslo-Vereinbarung vom 4. Dezember 1996 über einen definitiven Waffenstillstand zu verifizieren und Waffen von der Rebellenorganisation URNG einzusammeln und der Regierung zu übergeben. Danach wurde die rein zivile Mission fortgeführt. ${ }^{8}$

Wie u.a. MINUGUA zeigt, wandelten sich die humanitären Interventionen in der zweiten Hälfte der neunziger Jahre stark. Sie wurden schlanker, der militärische Teil wurde zurückgeschraubt und die Koordination verschiedener Massnahmen wurde in den Vordergrund gerückt. Während anfangs der neunziger Jahre viel vom «robusten peacekeeping» mit flankierenden zivilen Massnahmen die Rede war und sich - wie in Somalia - die zivil-militärischen Mandate vermischten, wird heute die Rolle des Militärs eher wieder subsidiär zu diplomatischen und humanitären Massnahmen gesehen - und sei es nur aus Kostengründen.

\section{$\square$ Anwachsen der humanitären Not- und Wiederaufbauhilfe}

Anders zeigt sich das Bild bei der humanitären Not- und Wiederaufbauhilfe. International sind die Ausgaben in diesem Bereich unverändert hoch und sogar im Anwachsen begriffen. Ein wesentlicher Grund dafür war und ist die Krise des peacekeeping, dem es in vielen Fällen nicht gelang, prekäre Waffenstillstände durchzusetzen, Gewalt in Konfliktgebieten einzudämmen und erzwungene Friedensvereinbarungen zu implementieren. Dadurch wurden Fragen über krisenadäquate Mandate aufgeworfen, Probleme der Überforderung des peacekeeping erörtet und das Verhältnis zwischen peacekeeping und «peace enforcement» auf die Tagesordnung gesetzt. ${ }^{9}$ Ausserdem wurde wiederholt der Verdacht geäussert, humanitäre Aktivitäten würden zunehmend als Ersatz für entschlossenes politisches Handeln betrachtet. ${ }^{10}$

Die Verlagerung vom internationalen peacekeeping zur humanitären Hilfe hat nämlich zur Folge, dass den Aufnahmestaaten im Inneren Kosten entstehen, welche die Mittel für die Nothilfe vor Ort oder sogar für eine peacekeepingOperation bei weitem übersteigen. Während letztere von der Gemeinschaft der Staaten gemäss einem Verteilerschlüssel getragen würden, werden die Kosten für die Flüchtlingsbetreuung einigen wenigen Ländern aufgebürdet. Die Verlagerung zur «post conflict rehabilitation» hat somit eine gewisse Entsolidarisierung in der internationalen Konfliktbewältigung zur Folge.

Der Fall Kosovo zeigt die Dilemmata humanitärer Aktivitäten deutlich auf. Während präventive Diplomatie als Mittel seit Anfang der neunziger Jahre zunächst gar nicht versucht wurde und nach Ausbruch der Krise wenig erfolgreich war, schien ein klassisches Mandat für eine peacekeeping-Mission (Blauhelme) vor dem Hintergrund der ausgebrochenen Kämpfe zwischen der Kosova-Befreiungsarmee UCK und paramilitärischen Kräften aus Belgrad wenig sinnvoll zu sein. Der Sicherheitsrat der Vereinten Nationen zeigte sich u.a. wegen des Widerstands von russischer Seite, vor allem aber wegen seiner

8. Findlay T. «Armed conflict prevention, management and resolution». In: Stockholm International Peace Research Institute (ed.), SIPRI Yearbook 1998. Armaments, Disarmament and International Security, Oxford University Press 1998, S. 40f.

9. Vgl. Kühne W. (Hrsg.) Blauhelme in einer turbulenten Welt, Baden-Baden (Nomos) 1993.

10. Eisele M. «Im Auftrag des Sicherheitsrates: Friedensmissionen der Vereinten Nationen». In: DGVN (Hrsg.) Vereinte Nationen Nr. 1 / Februar 1998 - 46. Jg., S. 6. 
restriktiven Mandatspolitik, äusserst zurückhaltend. Die NATO-Staaten wiederum zeigten wenig Begeisterung über einen möglichen Einsatz von Bodentruppen. Unter der ultimativen Drohung mit einem Luftschlag der NATO auf serbische Militäreinrichtungen wurde vom amerikanischen Unterhändler Richard Holbrooke mit dem serbischen Präsidenten Milosevic in letzter Minute ein Abkommen über einen Truppenabzug erzielt. Die wesentlichen politischen Probleme, die zur Krise geführt hatten, nämlich die Aufhebung der regionalen Autonomie durch Belgrad, konnten dadurch nicht gelöst werden.

Die darauf hin einsetzende humanitäre Hilfe wurde friedenspolitisch und ethisch begründet. Es war jedoch allen beteiligten Akteuren ebenfalls klar, dass angesichts des nahenden Wintereinbruchs die in die Wälder vertriebenen Dorfbewohner ins Ausland flüchten würden und dass die UCK durch eine Verschärfung der humanitären Krise weiter Auftrieb erhalten würde.

Das Abkommen mit Belgrad schaffte einerseits durchaus Raum für weitere diplomatische Schritte. Andererseits setzte es die OSZE-Mission und die humanitäre Not- und Wiederaufbauhilfe dem Risiko aus, in einem Gebiet zu operieren, das nicht oder nur gegen massive Widerstände pazifiziert worden ist. Die Entwicklung der politischen Lage ist daher Ende Oktober 1998 nur schwer abzuschätzen. Ein Aufflackern der Kämpfe ist nicht auszuschliessen. Und inwieweit die Drohung der NATO weiterhin wirksam bleibt, wenn humanitäre Aktivitäten vor Ort durchgeführt werden, bleibt ebenfalls eine offene Frage.

\section{$\square$ Humanitäre Hilfe in prekärem} sicherheitspolitischem Umfeld und als Teil von Kriegsökonomien

Die humanitäre Flüchtlingshilfe hängt wiederum unmittelbar von der Sicherheitslage in den jeweiligen Gebieten ab. Programme sind eher dort erfolgreich, wo existentielle Unsicherheiten aufgrund eines freiwilligen oder gut kontrollierten Friedensprozesses abgebaut werden konnten. Es gibt hingegen in denjenigen Gebieten Verzögerungen und Verhinderungen, in denen stagnierende Friedensprozesse eine Verlängerung der Nothilfe auf unbestimmte Zeit notwendig werden lassen. Dies zeigt die Konzentration der humanitären Mittel auf gewisse Krisengebiete, wie die Grossen Seen in Zentralafrika, das Horn von Afrika und Angola.

In einigen Gebieten ist die humanitäre Hilfe aufgrund zu hoher Sicherheits-risiken kaum oder nicht (mehr) möglich :

口 Im Nordkaukasus (Tschetschenien, Inguschetien, Nord-Ossetien) konnte wegen der äusserst prekären Sicherheitslage kaum humanitäre Hilfe an die Bevölkerung geleistet werden. Die Schweiz finanzierte lediglich die wenigen möglichen Aktivitäten von IKRK und UNHR mit;

口 Viele Regionen in Afrika verfügen nicht mehr über das Minimum an Sicherheit, das für die Durchführung humanitärer Mandate erforderlich ist (das gilt in ähnlicher Weise auch für Gebiete in Kolumbien). Im Gebiet der Grossen Seen wurden die Hilfsorganisationen trotz der massiven Nahrungsmitteldefizite aufgrund von Sicherheitsproblemen in ihren Aktivitäten stark limitiert. Eine substanzielle Demobilisierung der Armee wäre eine der notwendigen Voraussetzungen für eine Wiederaufbauhilfe, die in eine längerfristige Zusammenarbeit münden könnte. 
Ein weiterer sicherheitspolitischer Aspekt besteht im nicht zu unterschätzenden Problem, dass internationale Hilfeleistungen zum Bestandteil von Kriegs-ökonomien gemacht werden, so zum Beispiel durch

口 die Abschöpfung der Gelder grossangelegter Hilfsoperationen durch die bewusste Überbewertung der Währung des betroffenen Landes;

」 Abzweigung von Nahrungsmittelhilfe durch Bürgerkriegsparteien und durch von Flüchtlingen betroffene Staaten zur Versorgung von Truppen und Milizen;

\ Einsetzen der Nahrungsmittelhilfe als Waffe, indem diese oppositionsgeneigten Gruppen verwehrt wird;

๖ Verzerrung lokaler Macht- und Verteilungsstrukturen aufgrund des massiven Zuflusses von äusserer (Nahrungsmittel-)Hilfe."

Darüber hinaus klagen - vor allem NGOs mittlerer Grösse - über Korruption, die Erpressung von Schutzgeldern und andere Machenschaften von «warlords » oder schlicht kriminellen Banden.

\section{$\square$ Verlagerung humanitärer Aktivitäten auf die Nachkrisenzeit}

In Ländern mit stockenden Friedensprozessen wie in Angola, in akuten Bürgerkriegsgebieten mit wechselnder Intensität (Afghanistan, Kolumbien, Grosse Seen, Süd-Sudan, Somalia) und in Regionen mit Nachkrisensituationen (NordKaukasus) kann die humanitäre Hilfe der notleidenden Bevölkerung erst dann wirksam beistehen, wenn politische und militärische Kräfte zum Schutz der Aktivitäten mandatiert sind. Besteht eine Sicherheitslücke, sei es aufgrund erfolgloser präventiver Diplomatie, eines wirkungslosen oder nicht vorhandenen peacekeeping oder aufgrund eines nicht implementierten «peace enforcements », kann die humanitäre Nothilfe immer weniger auf dem Höhepunkt der Krise, sondern erst in einer Phase nach der Abkühlung des Konflikts geleistet werden. ${ }^{12}$

Dies ist nicht in jedem Fall negativ zu beurteilen. In Nachkrisenzeiten kann die humanitäre Hilfe unter Umständen wirkungsvoller und vor allem mit längerfristigen Erfolgen eingesetzt werden als unmittelbar in der heissen Phase. Dies ist dort der Fall, wo von der humanitären Nothilfe auf die ebenfalls humanitäre Wiederaufbauhilfe und die längerfristige Zusammenarbeit im Rahmen der EZA übergegangen werden kann. Eine gezielte Nachkrisen-intervention kann zur Prävention weiterer Krisen eingesetzt werden und damit unter Umständen mehr Menschenleben retten als reine Nothilfe unter prekären Bedingungen. Dies darf und kann jedoch nicht dazu führen, dass es wegen hoher Sicherheitsrisiken in der heissen Phase zur Unterlassung von Hilfe-leistungen kommt.

Schlussendlich hängen auch der Wirkungsgrad und die Nachhaltigkeit wesentlich von den Sicherheitslagen in den jeweiligen Gebieten ab. So kann es durchaus zum Stopp von Wiederaufbau oder Reintegration von Flüchtlingen aufgrund akuter Lagen kommen (Rwanda, Militärputsch in Sierra Leone). In BosnienHerzegowina sind Wiederaufbau und humanitäre Hilfe des verantwortlichen

11. Matthies V. (Hsg.) Vom Krieg zum Frieden, Kriegsbeendigung und Friedenskonsolidierung, Bremen 1995, S. 68f.

12. Vgl. Der Delegierte für humanitäre Hilfe und Chef SKH, Jahresbericht 1997 der Abteilung Humanitäre Hilfe und SKH, Bern im März 1998, S. 6. 
zivilen Peace Implementation Council nur unter dem Schutzschirm der SFROTruppen der NATO, die für die Sicherstellung des Abkommens von Dayton zuständig ist, überhaupt möglich geworden. Trotz dieser massiven militärischzivilen Mission kommt die Umsetzung des Friedensabkommens jedoch nur schleppend voran. So ist es für viele, die (freiwillig) zurückkehren möchten, unmöglich, an ihren Herkunftsort zurückzukehren, da ihre Sicherheit wegen ihrer ethnischen Zugehörigkeit nicht gewährleistet ist.

\section{$\square$ Gegenseitige Anpassung von humanitärer Hilfe und Sicherheitspolitik}

Fazit ist, dass sowohl die humanitäre Hilfe sich dem sicherheitspolitischen Umfeld anpassen muss, als auch die humanitäre Situation die Sicherheitspolitik verändert hat.

Mit der humanitären Begründung von Interventionen der Vereinten Nationen in Krisenregionen entfällt die Beliebigkeit und Interessengeleitetheit, wie sie dem peacekeeping der Vergangenheit anhafteten. Eine Triage lässt sich kaum humanitär begründen. Zur Gleichbehandlung humanitärer Krisen trägt darüber hinaus die zunehmende Verrechtlichung der Sicherheitspolitik bei. Sichtbarster Ausdruck dieser Entwicklung, die auf Rwanda und auf Bosnien zurückgeht, ist der in Rom beschlossene ständige Internationale Strafgerichtshof (International Criminal Court, ICC). Auch wenn seine Zuständigkeit und Reichweite noch nicht allen Erfordernissen einer Zeit mit inneren Konflikten und zerfallenden Staaten entspricht, so ist die Gerichtsbarkeit doch auf vier Kernverbrechen ausgedehnt, die heute für die wesentlichen humanitären Krisen verantwortlich sind: Völkermord, Verbrechen gegen die Menschlichkeit, Kriegsverbrechen und das Verbrechen des Angriffskriegs. ${ }^{13}$ Es bleibt abzuwarten, ob die vorgesehenen Auslösemechanismen zu einem Rückgang von kriegsbedingten humanitären Krisen führen werden.

Sollte der Sicherheitsrat bei seiner neuen Linie bleiben, ist in Zukunft höchst selten mit einem Mandat in der Grössenordnung der UNPROFOR im früheren Jugoslawien zu rechnen. Die Krise des peacekeeping wird eher dazu führen, dass die zivilen und humanitären Aktivitäten grössere Ausmasse annehmen werden - mit allen Gefahren, die damit verbunden sind.

Jüngstes Beispiel ist die Verifikationsmission der OSZE für den Kosovo (KVM). Die Mission, die aus 2000 unbewaffneten Zivil- und Militärpersonen besteht, setzt in dieser Hinsicht neue - aus der Not geborene - Massstäbe. Die KVM hat die Aufgabe, die Einhaltung des Waffenstillstandes, den Rückzug der jugoslawischen Sicherheitskräfte, die Rückkehr von Vertriebenen und Flüchtlingen und die Aufnahme von Verhandlungen zwischen Belgrad und Pristina zu überprüfen. Die KVM wird im weiteren humanitäre Organisationen unterstützen und die Durchführung von Wahlen in Kosovo überwachen. ${ }^{14}$ Insofern versteht sich die KVM als ziviler Beitrag zur Konfliktlösung, ein Beitrag, der jedoch vom militärisch erzeugten Druck der NATO abhängt.

13. Kaul H.P. «Der Internationale Strafgerichtshof: Das Ringen um seine Zuständigkeit und Reichweite». In: Humanitäres Völkerrecht $\mathrm{Nr} .3$, 1998, Themenheft Internationaler Strafgerichtshof, DRK, S. $138 \mathrm{ff}$.

14. Eidg. Departement für Auswärtige Angelegenheiten, Pressemitteilung: Schweizerische Beteiligung an der KosovoVerifikationsmission der OSZE, Bern, 21. Oktober 1998. 
Ob der Schutz der Menschen im Kosovo durch eine zivile humanitäre Mission gewährleistet werden kann, hängt von der politischen Stabilisierung ab. Und diese ist wiederum eine Funktion eines umfassenden Friedensprozesses, der sich nicht auf die Rückkehr von Vertriebenen und Flüchtlingen beschränken lässt.

\section{EIN SOLIDARITÄTSKORPS FÜR DIE HUMANITÄRE SICHERHEITSPOLITIK DER SCHWEIZ?}

Noch nie hat die Schweiz so viele Mittel in einem Krisengebiet ausgegeben wie im Konflikt in Ex-Jugoslawien. Insgesamt erreichten die Aufwendungen zwischen 1991 und 1996 einschliesslich der Hilfe für die Flüchtlinge in der Schweiz 1,2 Mrd. US\$. ${ }^{15}$

Die schweizerischen Beiträge zur Krisenbewältigung in Bosnien-Herzegowina umfassen Bereiche wie Finanzhilfe, humanitäre Hilfe, technische Zusammenarbeit, Kampagne für die Rückkehr der Flüchtlinge, Unterstützung des Wahlprozesses, Gelbmützen, Missionen der OSZE und Unterstützung unabhängiger Medien. Diese Vielfalt reflektiert den internationalen Trend insofern, als die Schweiz über kein eigenes peacekeeping-Kontingent verfügt und daher die humanitäre Hilfe in einem Verbund von anderweitigen Massnahmen wie unbewaffnete Militärbeobachter, Zivilpolizisten (CIVPOL), Einsätze des Katastrophenhilfekorps (SKH) und des Bundesamtes für Flüchtlinge (BFF), erfolgt. Zunehmendes Gewicht erhält seit dem Abschluss des Vertrags von Ottawa im Dezember 1997 die humanitäre Minenräumung und die Hilfe an die Minenopfer. Beiträge im Kampf gegen Minen stammen von der DEZA, dem Bundesamt für Flüchtlinge und dem Departement für Verteidigung, Bevölkerungsschutz und Sport (VBS).

Vor diesem Hintergrund ist der Bericht der Studienkommission für strategische Fragen ( Brunner-Bericht») konsequenterweise auf die solidarische Einbindung der Schweiz in internationale Sicherheitsstrukturen ausgerichtet. Im Bericht wird betont, dass direkte militärische Bedrohungen der Schweiz - wie auch ihrer Nachbarn - an Bedeutung verloren hätten. Schutz gegen die Risiken durch indirekte Auswirkungen von Krisen im geographischen Nah- und Fernbereich - wie in Bosnien oder im Kosovo - sei nur noch durch gleiche, unteilbare und zusammen mit anderen verantwortete Sicherheit zu erlangen. Die Kommission empfiehlt daher unter anderem die Bildung eines Schweizer Solidaritätskorps bestehend aus «besonders ausgebildetem und ausgerüstetem Berufs- und Milizpersonal (Freiwillige). Der Verband soll in der Lage sein, Rettungs- und Friedenseinsätze und Aktionen zur Unterstützung ziviler Behörden zu unternehmen » (Empfehlung Nr. 9).

Die Konsultationen zum Brunner-Bericht und auch Meinungsumfragen unter der Bevölkerung zeigen, dass eine humanitäre Sicherheitspolitik und selbst eine aktivere Rolle der Schweiz beim peacekeeping mehrheitlich mitgetragen würde. Nichtsdestotrotz wurde von verschiedenen Seiten die Frage aufgeworfen, ob das vorgeschlagene Solidaritätskorps im Rahmen des VBS wirklich das geeignete

15. Der Anteil der humanitären Hilfe an der gesamten öffentlichen EZA ist mit 19 bis $20 \%$ im internationalen Vergleich relativ hoch, blieb jedoch über die letzten zehn Jahre hinweg stabil. Vgl. IUED Jahrbuch Schweiz-Dritte Welt 1998, Nr. 17, Genf, April 1998, S. 323ff. 
Mittel sei, um den schweizerischen Beitrag an die internationale Friedens-förderung aufzupolieren. ${ }^{16}$

Vielfach wurde kritisiert, dass mit dem Solidaritätskorps auf künftige Bedrohungen nicht präventivpolitisch, sondern v.a. militärisch-technisch reagiert werde. So assoziiere man beim vorgeschlagenen Korps unweigerlich Wolldecken, Sanität, Transport, Logistik und Katastrophenhilfe, anstatt präventive Diplomatie, Kommunikation, Vermittlung und multilaterale Friedensförderung - Bereiche, welche dringend der Stärkung bedürfen $\cdot{ }^{17}$ Auch wurden Befürchtungen laut, das VBS würde mit dem Solidaritätskorps das bestehende Korps der SKH konkurrenzieren bzw. einverleiben wollen. Damit wird unweigerlich die Frage nach den Kosten und der Effizienz aufgeworfen, zumal das Verhältnis des zu schaffenden Korps mit den zahlreichen bereits bestehenden Aktivitäten (CIVPOL, Militärbeobachter, etc.) nicht klar herausgearbeitet wurde.

Zentral ist jedoch, dass ein Solidaritätskorps ins Leere stossen muss, wenn es nicht in politische Friedensbemühungen eingebettet und von Aktivitäten zur Krisenvorbeugung und -bewältigung eingerahmt ist. Will sich die Schweiz wirklich auf das inzwischen von kompetenten und mächtigen Akteuren besetzte Parkett der aktiven Friedensförderung begeben, braucht sie eine kohärente Strategie und keine vereinzelten «Operationen». Das Primat der Politik ist dabei ebenso wichtig wie im Inland. Präventive und vermittelnde Diplomatie - multilateral abgestimmt - ist die treibende Kraft für eine Teilnahme der Schweiz an internationalen Friedensbemühungen. Es liegt an der Aussenpolitik, diejenigen Krisenherde zu evaluieren, bei denen eine Anstrengung erfolgversprechend ist. Sie muss die dazu notwendigen Instrumente definieren und koordiniert zum Einsatz bringen. Das Solidaritätskorps könnte dabei allenfalls eine wichtige unterstützende Funktion wahrnehmen, ebenso wie die präventive Entwicklungszusammenarbeit, die Humanitäre Hilfe und die immer wichtiger werdenden Hilfestellungen von Nichtregierungsorganisationen. Ein solches Korps macht nur im Rahmen einer mit der Aussen- und Entwicklungspolitik sowie anderen Partnerstaaten abgestimmten Strategie der mittel- und langfristig konstruktiven Konfliktbearbeitung überhaupt Sinn.

\section{VON DER NOTHILFE ZUR PRÄVENTION}

International sind sich die Akteure darin einig, dass Prävention nur gelingen kann, wenn die knappen Mittel für die Friedenspolitik nicht für Nothilfe und Krisenintervention aufgezehrt werden.

An einer Konferenz der Vereinten Nationen zum Thema «Preventive Diplomacy: The Therapeutics of Mediation» in New York vom 23.-24. April 1996 verknüpfte Jan Eliasson, Staatssekretär im schwedischen Aussenministerium, die Überlebenschancen der Vereinten Nationen als wirkungsvolle Weltorganisation mit der Entwicklung erfolgversprechender präventiv-politischer Instrumente. Eliasson sprach von einem «präventiv- diplomatischen Imperativ» mit dem Ziel, «Milliarden von Dollars einzusparen, Menschenleben und die UNO

16. Vgl. VBS, Generalstab Kernteam Armee 200X (Hrsg.) Die Auswertung der Konsultation über den Bericht der Studienkommission für strategische Fragen (Bericht Brunner), Bern, 6.8.98.

17. So die Stellungnahme der MitarbeiterInnen des Instituts für Konfliktlösung der Schweizerischen Friedensstiftung. 
zu retten». Die Weltorganisation sei überfordert und erschöpft; sie befinde sich in einer schweren Finanz-, Identitäts- und Wertekrise. Zahlreiche und grosse Peacekeeping-Operationen könne sie sich nicht mehr leisten.

Um so entscheidender und zentraler im Instrumentarium der Vereinten Nationen zur Friedenssicherung ist daher der Bereich der viel weniger aufwendigen Präventivdiplomatie. Keine Frage, dass die friedenspolitische Vorsorge humaner, vernünftiger und erst noch viel kostengünstiger ist als Nachsorge, Konflikteindämmung und die Stationierung von Friedenstruppen. Wenn der Sicherheitsrat den oben erwähnten ständigen Strafgerichtshof ernst nimmt, kann er wesentlich zur Prävention von humanitären Krisen beitragen. Aufgrund der beschlossenen Mechanismen kann er Ländersituationen mit Verdachts-momenten in Richtung schwerster Verbrechen dem Gerichtshof nach Kap. VII der UN-Charta überweisen. Dies ist nur der rechtliche Aspekt von Prävention. Was darüber hinaus auf der Tagesordnung steht, ist ein politisches Konzept, das operationelle (kurzfristige) und strukturelle (langfristige) Prävention mit-einander verknüpft. ${ }^{18}$

Selbst wenn dieses Konzept einmal existieren und angewandt werden wird, ist davon auszugehen, dass die Krisenprävention auf absehbare Zeit nicht in jedem der zahlreichen Krisen und Konflikte mit Erfolg eingesetzt werden kann. Gescheiterte Prävention darf jedoch nicht dazu führen, dass direkt auf die humanitäre Hilfe als Notlösung zurückgegriffen wird. Vielmehr muss das peacekeeping aus seiner Krise befreit werden. Die gezogenen Lehren aus Bosnien, Somalia, Kosovo und anderen Schauplätzen sind bei künftigen humanitären Intervention zu berücksichtigen. Dem Sicherheitsrat bleibt zwischen UNPROFOR einerseits und MINUGUA andererseits eine Reihe von Handlungsoptionen. Die Erfahrung zeigt, dass das Krisen- und Konflikt-management in jeder Hinsicht politisch glaubwürdig sein muss. Folgende Maximen, die sich aus verschiedenen Diskussionssträngen resultieren, sollen dabei richtungsweisend sein:

口 Eine entschlossene Politik zur Vermeidung oder Linderung von Leid hat - wie die Nothilfe - mehr nach dem Gleichbehandlungsgrundsatz und weniger nach regionalen Präferenzen zu erfolgen.

口 Um humanitäres Unrecht zu ahnden, sollen die Motive der Drittpartei überparteilich und moralisch begründet sein.

- Die Mittel zur Erreichung des Ziels sollen verhältnismässig und problemadäquat sein. Peacekeeping und Nothilfe sind zu koordinieren, aber nicht $\mathrm{zu}$ vermischen.

- Eine humanitär begründete militärische Intervention soll erst erfolgen, wenn alle ernsthaften und mit ganzer Kraft unternommenen Versuche der Streitbeilegung erfolglos geblieben sind.

- Eine solche Intervention muss das kleinere Übel sein, das heisst, das aus der Intervention zu erwartende Wohl für die betroffenen Parteien und für die Weltgemeinschaft muss das zu erwartende Übel übersteigen.

- Eine humanitäre Intervention muss sowohl Erfolg als auch Opferschutz zum Ziel haben. Sie muss begrenzt sein, Ordnungsfunktion und daher quasi Polizeicharakter haben.

18. Vgl. u.a. Carnegie Commission on Preventing Deadly Conflict (ed.) Preventing Deadly Conflict. Final Report With Executive Summary, New York, December 1997; Report of The Aspen Institute Conference on Conflict Prevention: Strategies to Sustain Peace in the Post-Cold War World, July 30 - August 3, 1996, Aspen, Colorado. 
a Die moralische Gewissheit muss vorhanden sein, dass durch die Intervention schweres menschliches Leid gelindert bzw. verhindert werden kann.

In der Schweiz sind verschiedene Instrumente vorhanden, um den Kriterien zu genügen: DEZA, SKH, Flüchtlingshilfe, Friedensförderung, Menschenrechtsexpterten etc. Ein zusätzliches Solidaritätskorps müsste somit vor allem bestehende Lücken im Bereich des peacekeeping als Teil einer humanitären Intervention füllen.

Künftig könnte die geplante «Stiftung solidarische Schweiz» ein geeignetes Instrument zur gezielten Förderung einer politischen Kultur der Prävention sein. Durch ihre Aktivitäten zur Prävention von Armut und Marginalisierung, zur Vermeidung gewaltsamer Konflikte und zur Versöhnungsarbeit zwischen Konfliktparteien könnte sie gezielt dazu beitragen, bestehende Lücken im Spektrum der präventiven Politik zu schliessen. ${ }^{19}$

\begin{tabular}{ll}
\hline Akronyme & \\
BFF & Bundesamt für Flüchtlinge \\
BMZ & Bundesministerium für Zusammenarbeit (BRD) \\
CIVPOL & Civilian Police (United Nations) \\
FAST & $\begin{array}{l}\text { Früherkennung von Spannungen und Tatsachenermittlung } \\
\text { (Projekt Early Warning der Friedensstiftung) }\end{array}$ \\
ICC & International Criminal Court \\
KVM & Verifikationsmission der OSZE für den Kosovo \\
MINUGUA & United Nations Verification Mission in Guatemala \\
NATO & North Atlantic Treaty Organization \\
OECD & Organization for Economic Cooperation and Development \\
OSZE & Organisation für Sicherheit und Zusammenarbeit in Europa \\
SFOR & Stabilization force \\
SKH & Schweizerisches Katastrophenhilfekorps \\
UCK & Kosova-Befreiungsarmee \\
UNHCR & United Nations High Commissioner for Refugees \\
UNPA & United Nations Protected Area \\
UNPROFOR & United Nations Protection Force (Bosnien H.) \\
URNG & Unidad Revolucionaria Nacional Guatemalteca \\
\end{tabular}

19. Vgl. Eidg. Finanzdepartement, Gesetz über die «Stiftung solidarische Schweiz». Eröffnung des Vernehmlassungsverfahrens, Bern, im Juni 1998. 